PROCEEDINGS OF THE

AMERICAN MATHEMATICAL SOCIETY

Volume 129, Number 11, Pages 3303-3305

S 0002-9939(01)06012-9

Article electronically published on April 2, 2001

\title{
FOURIER RESTRICTION FOR AFFINE ARCLENGTH MEASURES IN THE PLANE
}

\author{
DANIEL M. OBERLIN
}

(Communicated by Christopher D. Sogge)

\begin{abstract}
We obtain an analog, uniform for a large class of curves in the plane, of the Fefferman-Zygmund theorem on restriction of the Fourier transform.
\end{abstract}

The purpose of this note is to prove an analog, uniform over a certain class of curves in $\mathbb{R}^{2}$, of the Fefferman-Zygmund restriction theorem for the circle.

Theorem. If $1 \leq p<\frac{4}{3}$ and $\frac{1}{p}+\frac{1}{3 q}=1$, there is a constant $C=C(p)$ such that the estimate

$$
\left(\int_{a}^{b}|\widehat{f}(t, \phi(t))|^{q} \phi^{\prime \prime}(t)^{\frac{1}{3}} d t\right)^{\frac{1}{q}} \leq C(p)\|f\|_{L^{p}\left(\mathbb{R}^{2}\right)}
$$

holds whenever $\phi$ is a real-valued function on an interval $(a, b)$ satisfying $\phi^{\prime \prime}(t)>0$, $\phi^{(3)}(t) \geq 0$ on $(a, b)$.

Writing $d \lambda$ for the measure $\phi^{\prime \prime}(t)^{1 / 3} d t$ on the curve $\gamma(t)=(t, \phi(t)), a<t<b$, we will follow the broad outline of the Fefferman-Zygmund proof and thus establish the dual estimate

$$
\|\widehat{f d \lambda}\|_{L^{q}\left(\mathbb{R}^{2}\right)} \leq C(p)\|f\|_{L^{p}(d \lambda)}
$$

where $1 \leq p<4$ and $\frac{1}{p}+\frac{3}{q}=1$.

Interpolation with the case $(p, q)=(1, \infty)$ shows that it is enough to prove a restricted weak type version of (2). Thus if $\frac{1}{p}=\frac{3}{2} \frac{1}{r}-\frac{1}{2}$, so that $\frac{1}{r}+\frac{2}{q}=1$, we will show that

$$
\left\|\left(\chi_{\gamma(E)} d \lambda\right) *\left(\chi_{\gamma(E)} d \lambda\right)^{\sim}\right\|_{L^{r, \infty}\left(\mathbb{R}^{2}\right)} \leq C(p)\left(\int_{E} \phi^{\prime \prime}(t)^{1 / 3} d t\right)^{\frac{2}{p}}
$$

whenever $E$ is a Borel subset of $(a, b)$. Then it will follow that

$$
\begin{gathered}
\left\|\widehat{\chi_{\gamma(E)} d} \lambda\right\|_{L^{q, \infty}\left(\mathbb{R}^{2}\right)}=\left\|\left.\widehat{\mid \chi_{\gamma(E)} d} \lambda\right|^{2}\right\|_{L^{\frac{q}{2}, \infty}}^{\frac{1}{2}} \\
\leq C(p)\left\|\left(\chi_{\gamma(E)} d \lambda\right) *\left(\chi_{\gamma(E)} d \lambda\right)^{\sim}\right\|_{\left.L^{r, \infty} \mathbb{R}^{2}\right)}^{\frac{1}{2}} \leq C(p)\left(\int_{E} \phi^{\prime \prime}(t)^{1 / 3} d t\right)^{\frac{1}{p}},
\end{gathered}
$$

Received by the editors March 15, 2000.

1991 Mathematics Subject Classification. Primary 42B10.

Key words and phrases. Fourier transform, restriction. 
by Hunt's generalization of the Hausdorff-Young theorem. Now (3) is true for all $(p, r)$ of interest if it is true for the two extreme cases $(p, r)=(1,1)$ and $(p, r)=$ $(4,2)$. The first of these is easy and so it is enough to establish the inequality

$$
\left\|\left(\chi_{\gamma(E)} d \lambda\right) *\left(\chi_{\gamma(E)} d \lambda\right)^{\sim}\right\|_{L^{2, \infty}\left(\mathbb{R}^{2}\right)} \leq C\left(\int_{E} \phi^{\prime \prime}(t)^{1 / 3} d t\right)^{\frac{1}{2}}
$$

for some absolute constant $C$. Inequality (4) may be regarded as a weak endpoint estimate for (the dual of) Fourier restriction. It is a consequence of

$$
\begin{aligned}
\int_{a}^{b} \int_{t}^{b} \chi_{T}(\gamma(t) & -\gamma(s)) \chi_{E}(s) \chi_{E}(t) \phi^{\prime \prime}(s)^{1 / 3} \phi^{\prime \prime}(t)^{1 / 3} d s d t \\
\leq & C\left(\int_{E} \phi^{\prime \prime}(t)^{1 / 3} d t\right)^{\frac{1}{2}}|T|^{1 / 2}
\end{aligned}
$$

where $|T|$ is the two-dimensional Lebesgue measure of an arbitrary Borel subset $T$ of $\mathbb{R}^{2}$. But the LHS of (5) is bounded by

$$
\left(\int_{a}^{b}\left(\int_{t}^{b} \chi_{T}(\gamma(t)-\gamma(s)) \phi^{\prime \prime}(s)^{1 / 3} d s\right)^{2} \phi^{\prime \prime}(t)^{1 / 3} d t\right)^{\frac{1}{2}}\left(\int_{E} \phi^{\prime \prime}(t)^{1 / 3} d t\right)^{\frac{1}{2}} .
$$

Thus it is enough to establish the inequality

$$
\int_{a}^{b}\left(\int_{t}^{b} \chi_{T}(\gamma(t)-\gamma(s)) \phi^{\prime \prime}(s)^{1 / 3} d s\right)^{2} \phi^{\prime \prime}(t)^{1 / 3} d t \leq 4|T| .
$$

Inequality (6) is (2) in $\mathrm{Q}$. We repeat the short proof for the reader's convenience.

The convexity of the graph of $\phi$ shows that the change of variables

$$
(s, t) \rightarrow \gamma(s)-\gamma(t)=(s-t, \phi(s)-\phi(t))
$$

is one-to-one. Thus

$$
\int_{a}^{b} \int_{a}^{b} \chi_{T}(\gamma(t)-\gamma(s))\left|\phi^{\prime}(s)-\phi^{\prime}(t)\right| d s d t \leq|T|
$$

and (6) will follow from the inequality

$$
\phi^{\prime \prime}(t)^{1 / 3}\left(\int_{t}^{b} \chi_{A}(s) \phi^{\prime \prime}(s)^{1 / 3} d s\right)^{2} \leq 4 \int_{t}^{b} \chi_{A}(s)\left(\phi^{\prime}(s)-\phi^{\prime}(t)\right) d s
$$

if $A \subseteq(t, b)$. To prove (7) we let $\left|A_{u}\right|$ stand for the (one-dimensional) Lebesgue measure of $A \cap(u, b)$ whenever $t \leq u \leq b$. Then

$$
\int_{t}^{b} \chi_{A}(s)\left(\phi^{\prime}(s)-\phi^{\prime}(t)\right) d s=\int_{t}^{b} \chi_{A}(s) \int_{t}^{s} \phi^{\prime \prime}(u) d u d s=\int_{t}^{b} \phi^{\prime \prime}(u)\left|A_{u}\right| d u .
$$

Also,

$$
\begin{aligned}
& \int_{t}^{b} \chi_{A}(s) \phi^{\prime \prime}(s)^{1 / 3} d s=\int_{t}^{b} \chi_{A}(s) \phi^{\prime \prime}(s)^{1 / 3}\left|A_{s}\right|^{1 / 3}\left|A_{s}\right|^{-1 / 3} d s \\
& \leq\left(\int_{t}^{b} \chi_{A}(s) \phi^{\prime \prime}(s)\left|A_{s}\right| d s\right)^{1 / 3}\left(\int_{t}^{b} \chi_{A}(s)\left|A_{s}\right|^{-1 / 2} d s\right)^{2 / 3} .
\end{aligned}
$$


Thus it follows from (8) that

$$
\begin{aligned}
& \left(\int_{t}^{b} \chi_{A}(s) \phi^{\prime \prime}(s)^{1 / 3} d s\right)^{3} \\
& \quad \leq\left(\int_{t}^{b} \chi_{A}(s)\left(\phi^{\prime}(s)-\phi^{\prime}(t)\right) d s\right)\left(\int_{t}^{b} \chi_{A}(s)\left|A_{s}\right|^{-1 / 2} d s\right)^{2} .
\end{aligned}
$$

If $0 \leq \rho \leq|A|$, then $\left|\left\{s \in A:\left|A_{s}\right| \leq \rho\right\}\right|=\rho$, and so

$$
\int_{t}^{b} \chi_{A}(s)\left|A_{s}\right|^{-1 / 2} d s=\int_{0}^{|A|} y^{-1 / 2} d y=2|A|^{1 / 2} .
$$

With this and the fact that $\phi^{\prime \prime}$ is nondecreasing, (9) yields (7) to complete the proof of the theorem.

The measure $d \lambda$ is called the affine arclength measure on the curve $\gamma$. Drury [D] was the first to point out its relevance to certain problems in harmonic analysis. In particular, [D] contains a restriction theorem using affine arclength measure on certain curves in $\mathbb{R}^{3}$.

\section{REFERENCES}

[D] S. W. Drury, Degenerate curves and harmonic analysis, Math. Proc. Camb. Phil. Soc. 108 (1990), 89-96. MR 91h:42021

[O] D. M. Oberlin, Convolution with affine arclength measures in the plane, Proc. Amer. Math. Soc. 127 (1999), 3591-3592. MR 2000c:42016

Department of Mathematics, Florida State University, Tallahassee, Florida 32306 4510

E-mail address: oberlin@math.fsu.edu 